\title{
Photo-physical properties of He-related color centers in diamond
}

\author{
G. Prestopino, ${ }^{1}$ M. Marinelli, ${ }^{1}$ E. Milani, ${ }^{1}$ C. Verona, ${ }^{1}$ G. Verona-Rinati, ${ }^{1}$ P. Traina, ${ }^{2}$ \\ E. Moreva ${ }^{2}$ I. P. Degiovanni, ${ }^{2}$ M. Genovese, ${ }^{2,4}$ S. Ditalia Tchernij, ${ }^{3,4}$ F. Picollo, ${ }^{3,4}$ \\ P. Olivero, ${ }^{3,4}$ and J. Forneris ${ }^{4,3, a)}$ \\ ${ }^{1}$ INFN-Dipartimento di Ingegneria Industriale, Università di Roma “Tor Vergata," Roma, Italy \\ ${ }^{2}$ Istituto Nazionale di Ricerca Metrologica (INRiM), Torino, Italy \\ ${ }^{3}$ Physics Department and "NIS" Inter-Departmental Centre, University of Torino, Torino, Italy \\ ${ }^{4}$ Istituto Nazionale di Fisica Nucleare (INFN) Sez. Torino, Torino, Italy
}

(Received 19 July 2017; accepted 31 August 2017; published online 13 September 2017)

\begin{abstract}
Diamond is a promising platform for the development of technological applications in quantum optics and photonics. The quest for color centers with optimal photo-physical properties has led in recent years to the search for novel impurity-related defects in this material. Here, we report on a systematic investigation of the photo-physical properties of two He-related (HR) emission lines at $535.2 \mathrm{~nm}$ and $559.7 \mathrm{~nm}$ (as measured at a temperature of $25 \mathrm{~K}$ ) created in three different diamond substrates upon implantation with $1.3 \mathrm{MeV} \mathrm{He}^{+}$ions and subsequent annealing. The spectral features of the HR centers were studied in an "optical grade" diamond substrate as a function of several physical parameters, namely, the measurement temperature, the excitation wavelength, and the intensity of external electric fields. The emission lifetimes of the $535.2 \mathrm{~nm}$ and $559.7 \mathrm{~nm}$ lines were also measured by means of time-gated photoluminescence measurements. The Stark shifting of the HR centers under the application of an external electrical field was observed in a CVD diamond film equipped with buried graphitic electrodes, suggesting the lack of inversion symmetry in the defects' structure. Furthermore, the photoluminescence mapping under $405 \mathrm{~nm}$ excitation of a "detector grade" diamond sample implanted at a $\mathrm{He}^{+}$ion fluence of $1 \times 10^{10} \mathrm{~cm}^{-2}$ enabled us to identify the spectral features of both the HR emission lines from the same localized optical spots. The reported results provide an insight into the structure of He-related defects in diamond and their possible utilization in practical applications. Published by AIP Publishing.

[http://dx.doi.org/10.1063/1.4996825]
\end{abstract}

Color centers in diamond are appealing physical systems for application in emerging quantum technologies. In recent years, growing interest in this research field led to the discovery, investigation, and fabrication of several classes of impurity-related defects. ${ }^{1-6}$

The formation of optically active defects in diamond upon He implantation followed by annealing in vacuum was recently reported. ${ }^{7}$ The spectral features of these defects consisted of two narrow emission lines at $536.5 \mathrm{~nm}$ and $560.5 \mathrm{~nm}$ at room temperature and a phonon sideband in the $572-630 \mathrm{~nm}$ spectral range. Following their initial observation in cathodoluminescence (CL), ${ }^{8,9}$ their optical activity was investigated in both photoluminescence (PL) and electroluminescence (EL). ${ }^{7,10}$

The need of understanding the possible formation of stable optically active centers, whose current (and still tentative) attribution is based on He incorporation in the diamond lattice, motivates a further investigation of their optophysical properties. More specifically, the centers have so far been attributed to the He-vacancy complex, ${ }^{11}$ and density functional theory calculations demonstrated that both this structure and the interstitial He defect could result in stable complexes in the diamond lattice. ${ }^{12}$ Apart from this limited body of works, a systematic set of experimental results on the opto-physical properties of He-related (hereafter referred to as HR) centers is still missing. Such results would be of

\footnotetext{
a)Author to whom correspondence should be addressed: forneris@to.infn.it
}

significant interest, as $\mathrm{He}$ implantation has been widely exploited for the fabrication of diamond-based optical micro-structures, ${ }^{13-15}$ under the implicit assumption of its chemical inertness in the diamond crystal. The understanding of the formation process and the emission properties of HR color centers could therefore enable us to either exploit them as possible single-photon emitters or to identify a suitable strategy to mitigate their undesired presence.

In this letter, we present an extensive characterization of single crystal diamond samples incorporating HR centers, with the purpose of gaining a deeper insight into their optophysical properties. Their PL spectral features were investigated as a function of temperature, excitation wavelength, and intensity of external electrical fields. Additionally, single-photon-sensitive PL confocal microscopy of a diamond substrate containing HR centers at very low densities enabled their preliminary mapping, thus providing indications on the spatial distribution/correlation of HR emission lines from the same complexes.

The reported measurements were performed on a set of three He-implanted samples: a type-IIa synthetic singlecrystal "optical grade" diamond plate $\left(3 \times 3 \times 0.3 \mathrm{~mm}^{3}\right.$ in size) by ElementSix (Sample \#1); a single-crystal diamond film grown at the laboratories of University of Rome "Tor Vergata" on a commercial high-pressure high-temperature (HPHT) Ib diamond substrate (Sample \#2); a type-IIa "detector grade" diamond plate by IIA Technologies (Sample $\# 3$ ) with a nominal substitutional $\mathrm{N}$ concentration of $<1 \mathrm{ppb}$. 
$500 \times 500 \mu \mathrm{m}^{2}$ regions were irradiated with a $1.3 \mathrm{MeV} \mathrm{He}{ }^{+}$ beam at fluences of $2 \times 10^{16} \mathrm{~cm}^{-2}$ and $1 \times 10^{10} \mathrm{~cm}^{-2}$ on Samples \#1 and \#3, respectively. Sample \#2 was fabricated with graphitic micro-electrodes by means of the deep ion beam lithography technique. ${ }^{16}$ Fabrication details are discussed in Refs. 10 and 16. To summarize, pairs of electrodes with a spacing of $\sim 12 \mu \mathrm{m}$ [see Fig. 3(a)] were fabricated through a $1.8 \mathrm{MeV} \mathrm{He}{ }^{+}$microbeam $\left(1.5 \times 10^{17} \mathrm{~cm}^{-2}\right.$ implantation fluence, i.e., above the graphitization threshold ${ }^{16}$ ). The utilization of a finite-size ion microbeam resulted in the creation of a "halo" of HR defects surrounding the electrodes, with an estimated implantation fluence of $\sim 10^{15} \mathrm{~cm}^{-2}$ at the center of the inter-electrode gap. All substrates were subsequently annealed at $1000^{\circ} \mathrm{C}$ for $2 \mathrm{~h}$ in vacuum to induce the formation of HR defects.

PL measurements as a function of temperature and excitation wavelength were performed on Sample \#1. The same experimental system described in Ref. 17 was used. Briefly, Sample \#1 was placed on a cold finger inside a double-stage cryostat, allowing to us vary its temperature in the $25-300 \mathrm{~K}$ range. A 3D movable micrometric stage enabled the positional control of the optical excitation (spot beam area $4.5 \times 10^{-4} \mathrm{~cm}^{2}$ ), which was in turn provided by a $5 \mathrm{~ns}$ pulsed laser $(20 \mathrm{~Hz}$ repetition rate) with a tunable wavelength in the $210-500 \mathrm{~nm}$ range. PL spectra were acquired using a spectrograph/gated multichannel-plate CCD camera system, enabling acquisitions with a time resolution of $2 \mathrm{~ns}$ (600 lines $\mathrm{mm}^{-1}$ and 1200 lines $\mathrm{mm}^{-1}$ gratings, respectively, which will be referred to as "low" and "high spectral resolution" modes in the following). Typical low-resolution PL spectra acquired with an excitation wavelength of $440 \mathrm{~nm}$ at $25 \mathrm{~K}$, $100 \mathrm{~K}, 200 \mathrm{~K}$, and $300 \mathrm{~K}$ are displayed in Figs. 1(a)-1(d), respectively. The decrease in the overall emission intensity at increasing temperatures is clear, as shown by the different scales adopted for the plots in Figs. 1(a)-1(d). The most prominent features at $25 \mathrm{~K}$ consist of the two HR emission lines at $535.2 \mathrm{~nm}$ (HR1) and $559.7 \mathrm{~nm}$ (HR2). The zerophonon line (ZPL) emission of the neutral nitrogen-vacancy center $\left(\mathrm{NV}^{0}\right)$ at $575 \mathrm{~nm}$ is also displayed for the sake of comparison. The relative intensities of the emission lines cannot be taken as a direct indication of their respective quantum efficiencies since the PL detection occurred with a fixed
70 ns delay with respect to the laser excitation, thus affecting the collected signals characterized by different emission lifetimes.

An additional emission doublet (not visible at roomtemperature, due to a higher PL background) consisted of two peaks centered at $562.7 \mathrm{~nm}$ and $563.3 \mathrm{~nm}$. This emission was previously reported in both PL and EL regimes ${ }^{18,19}$ in samples irradiated with $\mathrm{MeV} \mathrm{C}$ ions tentatively attributed to interstitial defects ${ }^{9,20}$ and cannot be therefore attributed to HR centers.

High-resolution spectra of the HR1 and HR2 lines, acquired at $100 \mathrm{~K}$ and normalized to the number of excitation photons per laser pulse [Figs. 1(e) and 1(f), black lines], did not exhibit any fine structure, within the $\sim 0.06 \mathrm{~nm}$ spectral resolution of the setup. Additional measurements carried out with an excitation wavelength of $220 \mathrm{~nm}$ [Figs. 1(e) and 1(f), red lines] demonstrate that the HR spectral features are active under excitation at shorter wavelengths. The higher emission intensity observed under $220 \mathrm{~nm}$ excitation is a consequence of the diamond band-to-band photon absorption, as shown in detail in Fig. 2(e).

The temperature dependence of the PL emission intensities is reported in Fig. 2(a), in which the integrated peak intensities after background subtraction are normalized to the respective values measured at $25 \mathrm{~K}$. The intensity decrease in the HR2 and $\mathrm{NV}^{0}$ emissions at increasing temperature is conveniently described by the higher probability for the radiative emission to involve excited vibrational states. Notably, in contrast, the HR1 emission intensity increases with the temperature, possibly indicating that such emission is associated with a phonon-assisted process.

Figure 2(b) shows the red-shift of HR1 (black filled squares) and HR2 (red filled circles) emission as a function of the fourth power of the temperature $\left(T^{4}\right)$, together with the same data for the $\mathrm{NV}^{0} \mathrm{ZPL}$ (blue filled triangles) for the sake of comparison. The reported features could not be ascribed to a temperature-dependent variation in the emission lifetime of the HR1 and HR2 emission; as in the $25-100 \mathrm{~K}$ range, they did not display any significant trend. The linearization of the energy shift dependence from $T^{4}$ is consistent with the consolidated interpretative model based on the contraction of the diamond lattice and on the electron-phonon coupling
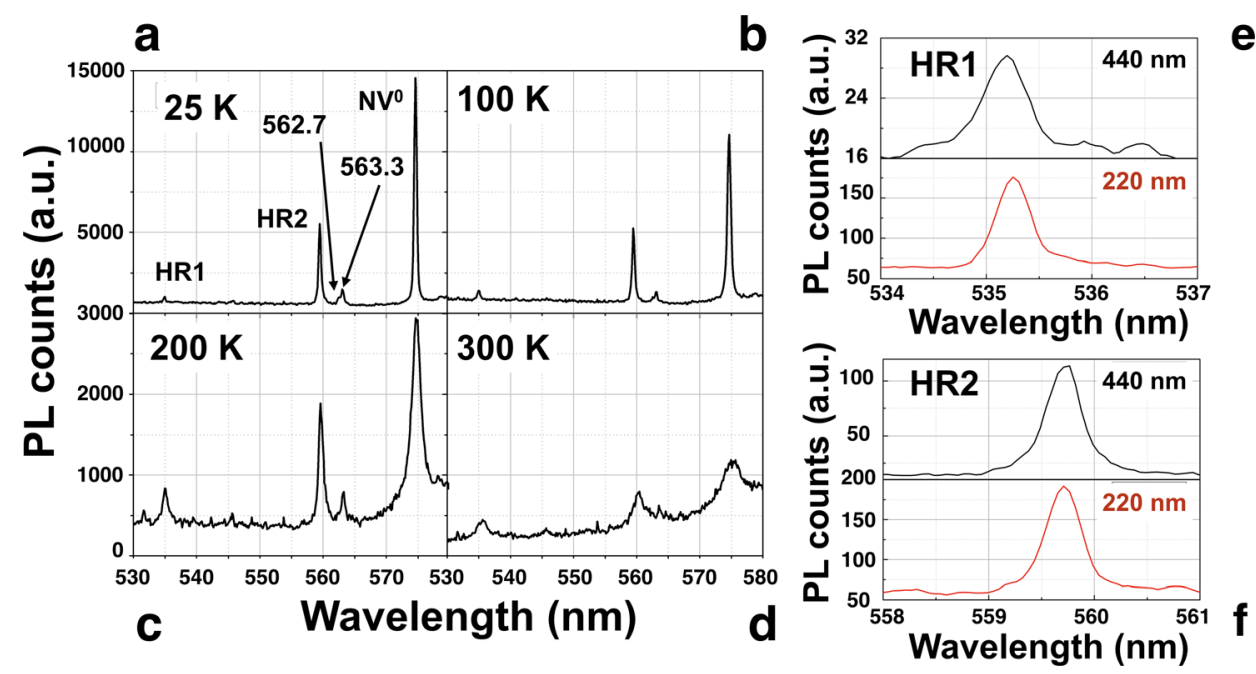

FIG. 1. PL spectra at different temperatures at an excitation wavelength of $440 \mathrm{~nm}$ : (a) $25 \mathrm{~K}$; (b) $100 \mathrm{~K}$; (c) $200 \mathrm{~K}$; (d) $300 \mathrm{~K}$. High resolution spectra of (d) HR1 and (e) HR2 emission lines measured at $100 \mathrm{~K}$ at excitation wavelengths of $440 \mathrm{~nm}$ (black lines) and $220 \mathrm{~nm}$ (red lines). 


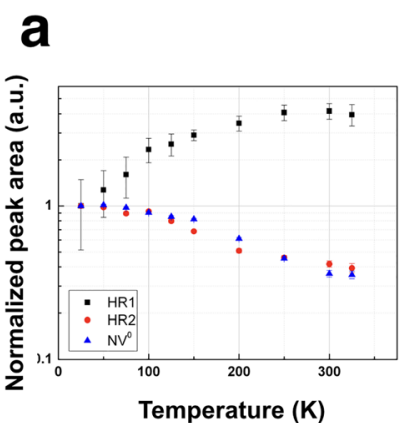

b
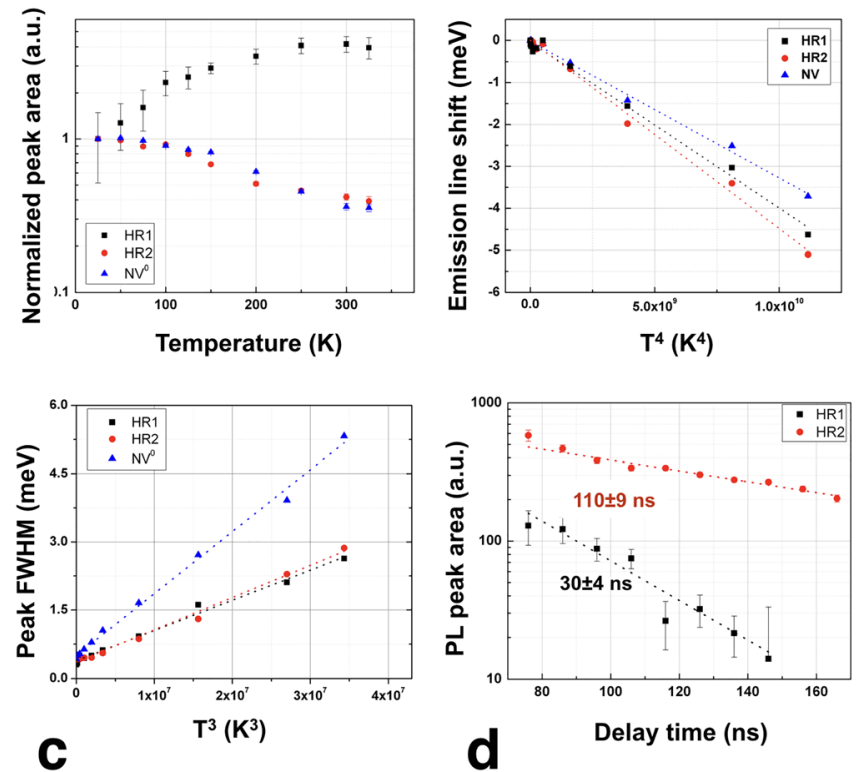

FIG. 2. (a) PL emission intensity of $\mathrm{HR} 1, \mathrm{HR} 2$, and $\mathrm{NV}^{0}$ centers as a function of temperature. Each set of data is normalized to the corresponding value measured at $25 \mathrm{~K}$. (b) Red-shift of the HR1, HR2, and $\mathrm{NV}^{0}$ lines as a function of $T^{4}$. (c) PL peak widths (FWHM) as a function of $T^{3}$. The dashed lines indicate the linear best fits. (d) Typical PL intensity decay chronograms of HR1 and HR2 emissions at $100 \mathrm{~K}$ under $440 \mathrm{~nm}$ laser excitation.

with hard phonon modes. ${ }^{9,21,22}$ The linear fitting procedure performed on the data in Fig. 2(b) provided slopes of $a_{\mathrm{HR} 1}=(-4.0 \pm 0.1) \times 10^{-10} \mathrm{meV} \mathrm{K}^{-4}$ and $a_{\mathrm{HR} 2}=(-4.5 \pm 0.1)$ $\times 10^{-10} \mathrm{meV} \mathrm{K}^{-4}$, for the HR1 and HR2 emission lines, respectively. For the sake of comparison, the dependence obtained for the NV center was $a_{\mathrm{NV} 0}=(-3.3 \pm 0.1) \times 10^{-10}$ $\mathrm{meV} \mathrm{K}^{-4}$, in line with previous reports. ${ }^{22}$

The dependence of the spectral widths (i.e., peak FWHM) of the HR1 and HR2 emissions on the third power of the temperature $\left(T^{3}\right)$ is reported in Fig. 2(c). PL data for the $\mathrm{NV}^{0}$ center are reported again for comparison. The linear

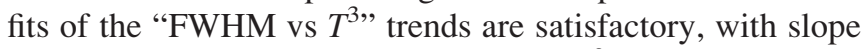
values for the HR1, HR2, and $\mathrm{NV}^{0}$ peaks of $b_{H R I}$ $=(6.6 \pm 0.2) \times 10^{-8} \mathrm{~nm} \mathrm{~K}^{-3}, \quad b_{H R 2}=(7.1 \pm 0.2) \times 10^{-8} \mathrm{~nm}$ $\mathrm{K}^{-3}$, and $b_{N V O}=(13.6 \pm 0.3) \times 10^{-8} \mathrm{~nm} \mathrm{~K}^{-3}$, respectively. This dependence is associated with the softening of the chemical bonds of the defect complex and results in a stronger coupling of its excited state with lattice phonons. ${ }^{23}$ The FWHM increase versus temperature is less pronounced for $\mathrm{HR}$ centers with respect to the $\mathrm{NV}^{0}$ emission, indicating that the former are characterized by a lower phonon coupling, as confirmed by their weaker phonon sidebands.

The PL emission lifetime of the HR1 and HR2 centers was estimated in Sample \#1 at a temperature of $100 \mathrm{~K}$ at an excitation wavelength of $440 \mathrm{~nm}(3.5 \mathrm{~mW}$ average power), by measuring the time-gated PL emission with respect to pulsed excitation. Typical PL intensity decay chronograms of the HR1 and HR2 emissions are reported in Fig. 2(d), together with the corresponding exponential fitting functions and the calculated decay times. From the statistical analysis of a set of $\sim 10$ independent measurements, the average emission lifetimes were estimated as $\tau_{\mathrm{HR} 1}=(29 \pm 5) \mathrm{ns}$ and $\tau_{\mathrm{HR} 2}=(106 \pm 10) \mathrm{ns}$ for the HR1 and HR2 emissions, respectively. These values were fully compatible with further measurements obtained under $220 \mathrm{~nm}$ excitation (not shown here).

The dependence of HR1 and HR2 PL intensity on the laser wavelength $(210-510 \mathrm{~nm}$ range; $100 \mathrm{~K}$ temperature) is shown in Fig. 3(a). The measured PL intensities were normalized to the number of wavelength-dependent photons per laser excitation pulse. The center position and the FWHM of the peaks did not display any significant variations over the considered spectral ranges. As an overall trend, HR1 and HR2 centers display decreasing PL emission intensities at increasing excitation wavelengths. An intensity maximum at $490 \mathrm{~nm}$ was apparent for the HR1 emission. Conversely, the HR2 line exhibited two maxima at 438 and $458 \mathrm{~nm}$. No significant mutual correlations between the HR1 and HR2 PL intensity variations were observed in the afore-mentioned range, and therefore, no possible associations with different charge states of the same complex could be inferred. Furthermore, no apparent center ionization, associated with a sudden decrease in the PL intensity at high energies, was observed down to the excitation wavelength of $405 \mathrm{~nm}$.

Remarkably, the data acquired under UV excitation revealed a sudden increase in the PL emission at wavelengths lower than $225 \mathrm{~nm}$ (corresponding to the band-edge absorption related to the $5.5 \mathrm{eV}$ band gap). This indicates that the recombination of electron-hole pairs generated upon band-to-band photon absorption in the UV provides a further excitation channel for the HR centers emission and thus confirms the role of the defects as electrically active traps in the material.

The Stark shifting of the HR centers under the application of external static electric fields was investigated on Sample \#2. PL spectra were acquired with the same setup described in Ref. 19 consisting of a Horiba Jobin Yvon HR800 Raman micro-spectrometer with a 1800 grooves $\mathrm{mm}^{-1}$ diffraction grating and a $532 \mathrm{~nm}$ laser excitation (21.6 $\mathrm{mW}$ power on the sample surface). A bias voltage difference in the $0-500 \mathrm{~V}$ range was applied to a small (i.e., $\sim 10 \times 15$ $\mu \mathrm{m}^{2}$ ) region comprised between sub-superficial graphitic electrodes. ${ }^{10}$ The position of the laser excitation spot $(\sim 3 \mu \mathrm{m}$ in size) is highlighted by the red circle in Fig. 3(b). It is worth remarking that the EL emission observed in previous works in the same regions ${ }^{10}$ was quenched by the intense laser excitation, which prevented the device from reaching the trap-filling-induced transition to the Poole-Frenkel conduction regime. ${ }^{18,19}$

The variations of the HR1 and HR2 PL spectra at increasing voltages are displayed in Figs. 3(c)-3(d). In Fig. $3(\mathrm{e})$, the spectral red-shift is reported against the bias voltage for the HR1 and HR2 emission lines. From a linear fitting of the data, it was found that the corresponding Stark shift effect was quantified with slope values of $A_{\mathrm{HR} 1}=-(1.6$ $\pm 0.8) \times 10^{-6} \mathrm{eV} \mathrm{V}^{-1}$ and $A_{\mathrm{HR} 2}=-(1.5 \pm 0.6) \times 10^{-6} \mathrm{eV}$ $\mathrm{V}^{-1}$, respectively. From these values, the local electric field $F$ was estimated according to the Lorentz local electric field approximation as $F=V(\varepsilon+2) / 2 d$, where $d=12 \mu \mathrm{m}$ is the electrode spacing, and the dielectric constant of $\varepsilon=5.7$ for pristine diamond was considered. ${ }^{24,25}$ The dipole moment change per unit of electric field $\Delta \mu$ could therefore be estimated from the expression $h \Delta \nu=-\Delta \mu \mathrm{F}+\mathrm{O}\left(\mathrm{F}^{2}\right),{ }^{24,25}$ where 
a

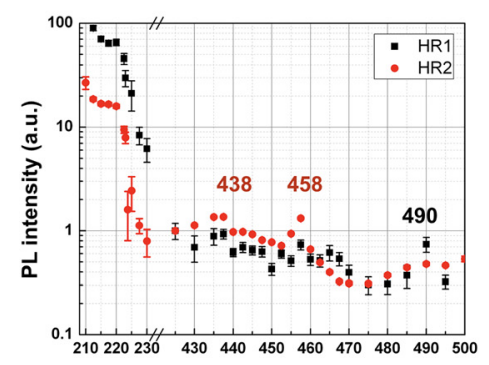

Excitation wavelength (nm)

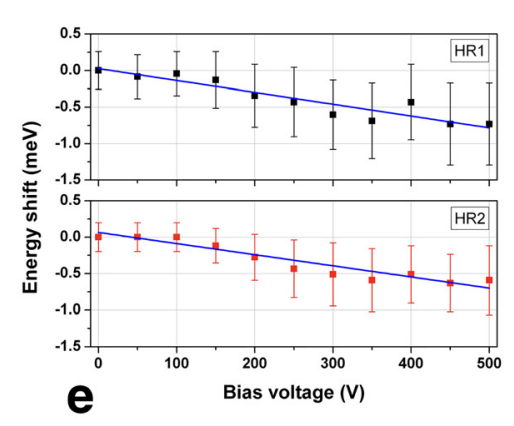

b
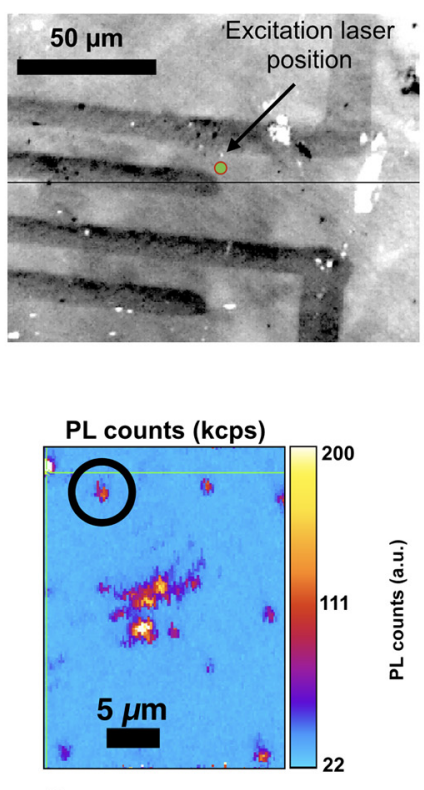

f
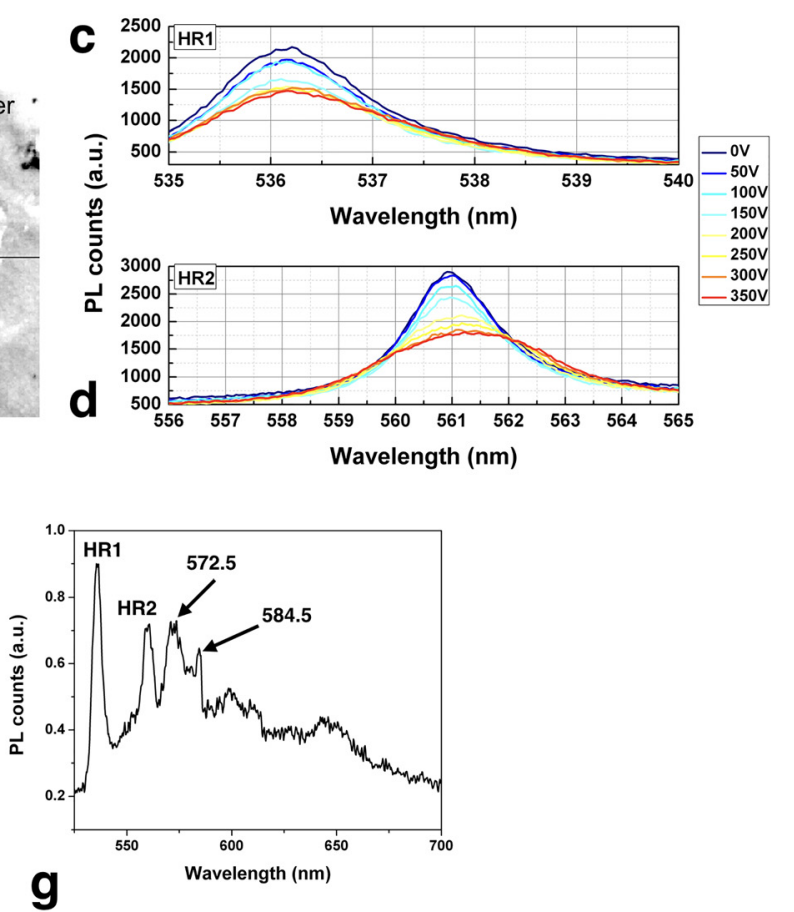

FIG. 3. (a) PL emission intensity at $100 \mathrm{~K}$ from HR1 and HR2 centers as a function of laser excitation wavelength in the $210-510$ nm range. (b) Optical micrograph of the graphitic micro-electrodes in Sample \#2. The red circle indicates the position of the laser excitation spot. PL spectra of the (c) HR1 center and (d) HR2 center under increasing applied bias voltage. (e) Red shift in the HR1 and HR2 emission at increasing bias. The blue lines indicate the respective linear best fits. (f) Typical PL map acquired from Sample \#3 under $405 \mathrm{~nm}$ laser excitation. (g) PL spectrum acquired from the spot circled in black in (f).

the second-order term was neglected consistent with the linear model adopted for the data fitting. The value of $\Delta \mu$ was quantified through the expression $\Delta \mu=-A \cdot 2 d /(\varepsilon+2)$ to be $(5 \pm 3) \times 10^{-12} \mathrm{eV} \mathrm{m} \mathrm{V}{ }^{-1}$ for both HR1 and HR2 emissions, corresponding to $0.24 \pm 0.15 \mathrm{D}$ (debye). This value is significantly smaller than that found for the NV center in diamond (i.e., $>1 \mathrm{D}^{25}$ ) as expected considering the physical volume of HR impurities.

The observation of a Stark shift of the HR1 and HR2 centers indicates the lack of inversion symmetry in the defect structure, ${ }^{11}$ in agreement with the interpretation of the emission as associated with $\mathrm{He}-\mathrm{V}$ defects rather than with interstitial He. ${ }^{9,11,12}$

Confocal PL mapping of HR defects was performed on Sample \#3 under $405 \mathrm{~nm}$ CW laser excitation (15 mW power at the sample surface) using a single-photon-sensitive setup ${ }^{7}$ with photon collection limited by a set of filters to the 500-570 nm spectral range. PL spectra were acquired through a single-grating monochromator (1600 grooves $\mathrm{mm}^{-1}$, $600 \mathrm{~nm}$ blaze). The sample, containing a low density of both irradiation-induced HR and native NV centers, was investigated to gain an insight into the spatial distribution and mutual correlations of HR1 and HR2 emissions. A typical confocal PL map is presented in Fig. 3(f). It exhibits several bright isolated spots with a size compatible with that of isolated emitters embedded among larger luminescent regions, while the substrate is characterized by a low fluorescence background. Remarkably, all the above-mentioned regions (i.e., both the isolated spots and the larger agglomerates) have similar spectral features. A typical PL spectrum from one of these spots [circled in black in Fig. 3(f)] is shown in Fig. 3(g).

Besides the HR1 and HR2 lines, the spectrum displays additional spectral features, such as two PL peaks at
$572.5 \mathrm{~nm}$ and $584.5 \mathrm{~nm}$, together with the HR2 phonon replica in the $580-630 \mathrm{~nm}$ range. ${ }^{7}$ The present measurements performed on a substrate with a low concentration of $\mathrm{NV}$ centers suggest that the origin of such spectral features is related to the HR emissions. Despite the fact that preliminary measurements of the second-order autocorrelation function ${ }^{26}$ did not enable us to identify single-photon emission, our point measurements indicate that both HR1 and HR2 emissions come from the same spot, i.e., possibly from the same defect complex. ${ }^{8}$ On the other hand, the PL emission from the isolated spots did not exhibit any intensity variations over the $\sim 500$ s measurement duration. Therefore, at least at these spatial (i.e., $\sim 1 \mu \mathrm{m})$ and temporal $\left(\sim 10^{-1} \mathrm{~s}\right)$ resolutions, the attribution of the HR1 and HR2 emissions to different charge states of the same defect could not be directly verified. The HR1 emission at $535.2 \mathrm{~nm}$ could be regarded as the center's ZPL, while the attribution of the HR2 emission is compatible with a quasi-local vibrational replica at an energy shift of $102 \mathrm{meV} .{ }^{9}$ Consistently, the $572.5 \mathrm{~nm}$ and $584.5 \mathrm{~nm}$ emissions could be ascribed to the ZPL interaction with optical phonons (150 meV and $196 \mathrm{meV}$, respectively). This interpretation is consistent with the inability to spatially distinguish the HR1 and HR2 in the confocal mapping experiments. On the other hand, the broadening and PL intensity increase of the HR1 line with the temperature might not be in full agreement with this interpretation. Another possible attribution consists of assigning the ZPL emission to the HR2 line $(559.7 \mathrm{~nm})$, having a temperature dependence similar to the $\mathrm{NV}^{0}$ ZPL. Accordingly, the HR1 emission increase with the temperature could indicate a blueshifted replica of HR2 involving the absorption (rather than the generation) of a quasi-local lattice vibration $(102 \mathrm{meV})$. The $572.5 \mathrm{~nm}$ and $584.5 \mathrm{~nm}$ lines and the band centered at 
$\sim 600 \mathrm{~nm}$ would then correspond to quasi-local vibrations of $50 \mathrm{meV}, 92 \mathrm{meV}$, and $\sim 150 \mathrm{meV}$ with respect to the HR2 ZPL. While this tentative model has the advantage of providing a clear attribution to the emission features at $>600 \mathrm{~nm}$, its reliability might be questioned by the considerable sharpness and intensity of the HR1 line.

We presented a detailed characterization of the photophysical properties of HR center ensembles fabricated in diamond upon $\mathrm{MeV} \mathrm{He}$ implantation. The centers did not exhibit any fine structure at temperatures as low as $25 \mathrm{~K}$, and their relevant emission features (emission lines shift, peaks FWHM) followed the typical trend against the temperature of stable defects in the diamond lattice. In contrast to the HR2 line, we observed a PL increase of the HR1 emission intensity at increasing temperatures, which could indicate the presence of a phonon-assisted radiative process.

The HR centers could be effectively excited under both visible and UV radiation and exhibited intense luminescence at energies higher than the diamond band gap, thus confirming their activity in CL and EL regimes. The observation of a Stark shift effect under applied electrical bias ruled out the inversion symmetry from their defect structure. Finally, PL confocal mapping under $405 \mathrm{~nm}$ excitation revealed that the HR1 and HR2 emissions arise from the very same luminescent spots in the sample. Future studies will investigate the HR emission at the single-photon emitter level to further clarify their attribution.

In principle, the HR1 emission lifetime $\left[\tau_{\mathrm{HR} 1}=(29 \pm 5)\right.$ ns at $100 \mathrm{~K}]$ should enable the detection of luminescence from individual defects, while the HR2 line is almost three times weaker in emission due to its relatively long lifetime $\left[\tau_{\mathrm{HR} 2}=(106 \pm 10) \mathrm{ns}\right]$. Overall, the narrow emission of HR centers, their small phonon coupling, and their photoexcitability in a wide spectral range, combined with the large availability of focused He beams for the centers' fabrication, ${ }^{27-29}$ provide a promising perspective on the application of HR defects in quantum technologies.

This research activity was supported by the following projects: "DIESIS" project funded by the Italian National Institute of Nuclear Physics (INFN)—CSN5 within the "Young research grant" scheme, Coordinated Research Project F11020 funded by the International Atomic Energy Agency (IAEA), and Progetto Premiale 2014 "QSecGroundSpace" funded by MIUR. MeV He implantations were performed within the "Dia.Fab." experiment at the INFN-LNL laboratories.

${ }^{1}$ J. O. Orwa, A. D. Greentree, I. Aharonovich, A. D. C. Alves, J. Van Donkelaar, A. Stacey, and S. Prawer, J. Lumin. 130, 1646 (2010).

${ }^{2}$ S. Pezzagna, D. Rogalla, D. Wildanger, J. Meijer, and A. Zaitsev, New J. Phys. 13, 035024 (2011).

${ }^{3}$ I. Aharonovich, S. Castelletto, D. A. Simpson, C.-H. Su, A. D. Greentree, and S. Prawer, Rep. Prog. Phys. 74, 076501 (2011).
${ }^{4}$ T. Iwasaki, F. Ishibashi, Y. Miyamoto, Y. Doi, S. Kobayashi, T. Miyazaki, K. Tahara, K. D. Jahnke, L. J. Rogers, B. Naydenov, F. Jelezko, S. Yamasaki, S. Nagamachi, T. Inubushi, N. Mizuochi, and M. Hatano, Sci. Rep. 5, 12882 (2015).

${ }^{5}$ D. Gatto Monticone, P. Traina, E. Moreva, J. Forneris, P. Olivero, I. P. Degiovanni, F. Taccetti, L. Giuntini, G. Brida, G. Amato, and M. Genovese, New J. Phys. 16, 053005 (2014).

${ }^{6}$ R. John, J. Lehnert, M. Mensing, D. Spemann, S. Pezzagna, and J. Meijer, New J. Phys. 19, 053008 (2017).

${ }^{7}$ J. Forneris, A. Tengattini, S. D. Tchernij, F. Picollo, A. Battiato, P. Traina, I. P. Degiovanni, E. Moreva, G. Brida, V. Grilj, N. Skukan, M. Jakšic', M. Genovese, and P. Olivero, J. Lumin. 179, 59 (2016).

${ }^{8}$ V. D. Tkachev, A. M. Zaitsev, and V. V. Tkachev, Phys. Status Solidi. B 129, 129 (1985).

${ }^{9}$ A. M. Zaitsev, Optical Properties of Diamond (Springer, New York, 2001).

${ }^{10}$ J. Forneris, A. Battiato, D. Gatto Monticone, F. Picollo, G. Amato, L. Boarino, G. Brida, I. P. Degiovanni, E. Enrico, M. Genovese, E. Moreva, P. Traina, C. Verona, G. Verona Rinati, and P. Olivero, Nucl. Instrum. Methods B 348, 187 (2015).

${ }^{11}$ B. Dischler, Handbook of Spectral Lines in Diamond Vol.1: Tables and Interpretations (Springer, Berlin, 2012).

${ }^{12}$ J. P. Goss, R. J. Eyre, and P. R. Briddon, Phys. Rev. B 80, 085204 (2009).

${ }^{13}$ F. Fávaro de Oliveira, S. A. Momenzadeh, D. Antonov, J. Scharpf, C. Osterkamp, B. Naydenov, F. Jelezko, A. Denisenko, and J. Wrachtrup, Nano Lett. 16(4), 2228 (2016).

${ }^{14}$ P. Olivero, S. Rubanov, P. Reichart, B. Gibson, S. Huntington, J. Rabeau, A. Greentree, J. Salzman, D. Moore, D. Jamieson, and S. Prawer, Adv. Mater. 17, 2427 (2005).

${ }^{15}$ I. Bayn, B. Meyler, A. Lahav, J. Salzman, R. Kalish, B. A. Fairchild, S. Prawer, M. Barth, O. Benson, T. Wolf, P. Siyushev, F. Jelezko, and J. Wrachtrup, Diamond Relat. Mater. 20, 937 (2011).

${ }^{16}$ J. Forneris, V. Grilj, M. Jakšic', A. Lo Giudice, P. Olivero, F. Picollo, N. Skukan, C. Verona, G. Verona-Rinati, and E. Vittone, Nucl. Instrum. Methods B 306, 181 (2013).

${ }^{17}$ M. G. Donato, G. Messina, G. Verona Rinati, S. Almaviva, G. Faggio, M. Marinelli, E. Milani, G. Prestopino, S. Santangelo, P. Tripodi, and C. Verona, J. Appl. Phys. 106, 053528 (2009).

${ }^{18}$ J. Forneris, P. Traina, D. Gatto Monticone, G. Amato, L. Boarino, G. Brida, I. P. Degiovanni, E. Enrico, E. Moreva, V. Grilj, N. Skukan, M. Jakšic', M. Genovese, and P. Olivero, Sci. Rep. 5, 15901 (2015).

${ }^{19}$ J. Forneris, S. Ditalia Tchernij, A. Tengattini, E. Enrico, V. Grilj, N. Skukan, G. Amato, L. Boarino, M. Jakšic', and P. Olivero, Carbon 113, 76 (2017).

${ }^{20}$ J. W. Steeds, S. Charles, T. J. Davis, A. Gilmore, J. Hayes, D. Pickard, and J. E. Butler, Diamond Relat. Mater. 8, 94 (1999).

${ }^{21}$ K. Dragounova, Z. Potucek, S. Potocky, Z. Bryknar, and A. Kromka, J. Electron. Eng. 68, 74 (2017).

${ }^{22}$ M. W. Doherty, V. M. Acosta, A. Jarmola, M. S. J. Barson, N. B. Manson, D. Budker, and L. C. L. Hollenberg, Phys. Rev. B 90, 041201 (2014).

${ }^{23}$ V. Hizhnyakov, H. Kaasik, and I. Sildos, Phys. Status Solidi. B 234, 644 (2002).

${ }^{24}$ T. Müller, I. Aharonovich, L. Lombez, Y. Alaverdyan, A. N. Vamivakas, S. Castelletto, F. Jelezko, J. Wrachtrup, S. Prawer, and M. Atatüre, New J. Phys. 13, 075001 (2011).

${ }^{25}$ P. Tamarat, T. Gaebel, J. R. Rabeau, M. Khan, A. D. Greentree, H. Wilson, L. C. L. Hollenberg, S. Prawer, P. Hemmer, F. Jelezko, and J. Wrachtrup, Phys. Rev. Lett. 97, 083002 (2006).

${ }^{26}$ A. Beveratos, S. Kühn, R. Brouri, T. Gacoin, J.-P. Poizat, and P. Grangier, Eur. Phys. J. D 18, 191 (2002).

${ }^{27}$ A. H. Piracha, K. Ganesan, D. W. M. Lau, A. Stacey, L. P. McGuinness, S. Tomljenovic-Hanic, and S. Prawer, Nanoscale 8, 6860 (2016).

${ }^{28}$ Z. Huang, W.-D. Li, C. Santori, V. M. Acosta, A. Faraon, T. Ishikawa, W. Wu, D. Winston, R. S. Williams, and R. G. Beausoleil, Appl. Phys. Lett. 103, 081906 (2013).

${ }^{29}$ D. McCloskey, D. Fox, N. O’Hara, V. Usov, D. Scanlan, N. McEvoy, G. S. Duesberg, G. L. W. Cross, H. Z. Zhang, and J. F. Donegan, Appl. Phys. Lett. 104, 031109 (2014). 